\title{
High-performance nanostructured thermoelectric materials
}

\author{
Jing-Feng Lii ${ }^{*}$, Wei-Shu Liu ${ }^{1,2}$, Li-Dong Zhao ${ }^{1,2}$ and Min Zhou ${ }^{1,3}$ \\ Tsinghua University, China
}

\begin{abstract}
Thermoelectric effects enable direct conversion between thermal and electrical energy and provide an alternative route for power generation and refrigeration. Over the past ten years, the exploration of high-performance thermoelectric materials has attracted great attention from both an academic research perspective and with a view to industrial applications. This review summarizes the progress that has been made in recent years in developing thermoelectric materials with a high dimensionless figure of merits (ZT) and the related fabrication processes for producing nanostuctured materials. The challenge to develop thermoelectric materials with superior performance is to tailor the interconnected thermoelectric physical parameters - electrical conductivity, Seebeck coefficient and thermal conductivity - for a crystalline system. Nanostructures provide a chance to disconnect the linkage between thermal and electrical transport by introducing some new scattering mechanisms. Recent improvements in thermoelectric efficiency appear to be dominated by efforts to reduce the lattice thermal conductivity through nanostructural design. The materials focused in this review include $\mathrm{Bi}-\mathrm{Te}$ alloys, skutterudite compounds, $\mathrm{Ag}-\mathrm{Pb}-\mathrm{Sb}-\mathrm{Te}$ quaternary systems, half-Heusler compounds and some high-ZT oxides. Possible future strategies for developing thermoelectric materials are also discussed.
\end{abstract}

T he conversion of thermal energy to electrical energy is known as thermoelectric (TE) conversion. The TE effect can be used for both power generation and electronic refrigeration. When a temperature gradient $(\Delta T)$ is applied to a TE couple consisting of n-type (electron-transporting) and p-type (hole-transporting) elements, the mobile charge carriers at the hot end tend to diffuse to the cold end, producing an electrostatic potential $(\Delta V)$. This characteristic, known as the Seebeck effect, where $\alpha=\Delta V / \Delta T$ is defined as the Seebeck coefficient, is the basis of TE power generation, as shown in Figure 1(a). Conversely, when a voltage is applied to a TE couple, the carriers attempt to return to the electron equilibrium that existed before the current was applied by absorbing energy at one connector and releasing it at the other, an effect known as the Peltier effect, as shown in Figure 1(b). Thermoelectric technology and solid-state devices based on the TE effect have a number of advantages, including having no moving parts, and being reliable and scalable. The technology has therefore arouse worldwide interest in many fields, including waste heat recovery and solar heat utilization (power generation mode), and temperature-controlled seats, portable picnic coolers and thermal management in microprocessors (active refrigeration mode) [1].

The efficiency of TE devices is strongly associated with the dimensionless figure of merit $(Z T)$ of TE materials, defined as $Z T=\left(\alpha^{2} \sigma / \kappa\right) T$, where $\sigma, \kappa$ and $T$ are the electrical resistivity, thermal conductivity and absolute temperature [2]. High electrical conductivity (corresponding to low Joule heating), a large Seebeck coefficient (corresponding to large potential difference) and low thermal conductivity (corresponding to a large temperature difference) are therefore necessary in order to realize high-performance TE materials. The $Z T$ figure is also a very convenient indictor for evaluating the potential efficiency of TE devices. In general, good TE materials have a $Z T$ value of close to unity. However, $Z T$ values of up to three are considered to be essential for
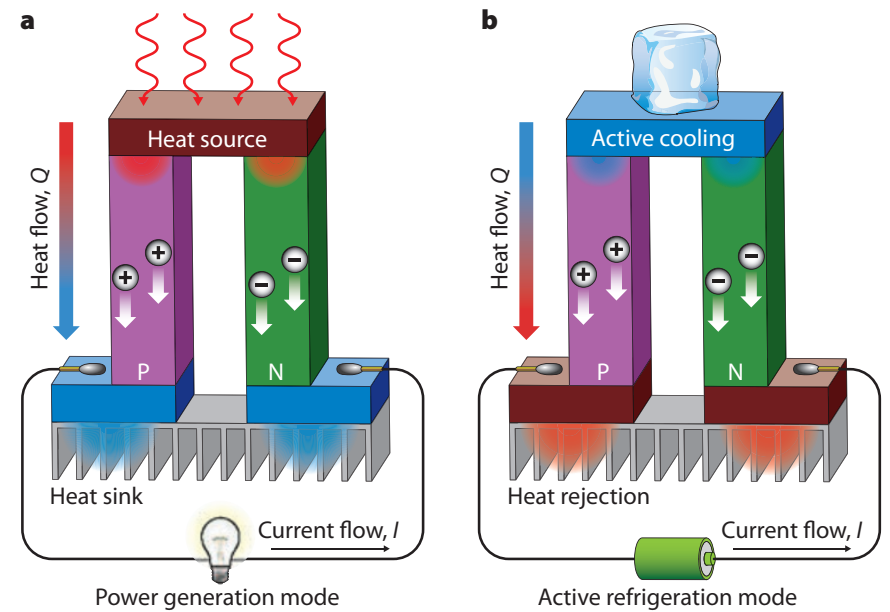

Figure 1. Schematic illustrations of a thermoelectric module for (a) power generation (Seebeck effect) and (b) active refrigeration (Peltier effect). (a) An applied temperature difference causes charge carriers in the material (electrons or holes) to diffuse from the hot side to the cold side, resulting in current flow through the circuit. (b) Heat evolves at the upper junction and is absorbed at the lower junction when a current is made to flow through the circuit.

TE energy converters that can compete on efficiency with mechanical power generation and active refrigeration.

High-performance TE materials have been pursued since $\mathrm{Bi}_{2} \mathrm{Te}_{3}$ based alloys were discovered in the 1960s. Until the end of last century, moderate progress had been made in the development of TE materials. The benchmark of $Z T \approx 1$ was broken in the mid-1990s by two

\footnotetext{
*Corresponding author. Email: jingfeng@mail.tsinghua.edu.cn

1. State Key Laboratory of New Ceramics and Fine Processing, Department of Materials Science and Engineering, Tsinghua University, Beijing 100084, China

2. School of Materials Science and Engineering, University of Science and Technology Beijing, Beijing, China

3. Technical Institute of Physics and Chemistry, Chinese Academy of Sciences, Beijing, China
} 
different research approaches: one by exploring new materials with complex crystalline structures, and the other by reducing the dimensions of the materials. The search for new materials, such as skutterudites $[3,4]$ and clathrates [5], is mainly motivated by the suggestion of Slack [6]. In those compounds, the 'rattling' motion of loosely bonded atoms within a large cage generates strong scattering against lattice phonon propagation, but has less of an impact on the transport of electrons. As a consequence, the thermal conductivity of skutterudites and clathrates can be reduced greatly while maintaining the electrical conductivity at a high level. Zintl compounds [7] with a large unit cell, including $\mathrm{Yb}_{14} \mathrm{MnSb}_{11}, \mathrm{Yb}_{11} \mathrm{GaSb}_{9}, \mathrm{Ca}_{11} \mathrm{GaSb}_{9}$ and $\mathrm{SrZnSb}_{2}$ have also been revealed to possess an intrinsically low lattice thermal conductivity due to the high fraction of low-velocity optical phonon modes [8]. In research on low-dimensional material systems, Dresselhaus et al. [9] suggested that the power factor $\left(\alpha^{2} \sigma\right)$ can be enhanced through the use of quantumconfinement effects. As the system size decreases and approaches a nanometer length scale, the density of electronic states (DOS) can split and become narrow. Dresselhaus's pioneering work has shed light on various low-dimensional systems, including superlattices, nanowires and quantum dots. Venkatasubramanian et al. [10] reported $\mathrm{Bi}_{2} \mathrm{Te}_{3} / \mathrm{Sb}_{2} \mathrm{Te}_{3}$ superlattices with a high- $Z T$ value of up to 2.4. Subsequently, Harman et al. [11] reported $\mathrm{PbTe} / \mathrm{PbTeSe}$ quantum dot superlattices with a $Z T$ value of greater than 3.0 at $600 \mathrm{~K}$. However, for general applications, it is highly desired to develop high-performance bulk TE materials. Figure 2 shows the high-performance bulk TE materials developed recently on the basis of nanostructural concepts, demonstrating the significant progress that has been made in TE materials since the introduction of nanotechnology. This review covers the latest advancements in TE technology focusing on such nanostructural approaches, and in doing so aims to highlight the potential routes for improvement in the major TE material systems, including $\mathrm{Bi}-\mathrm{Te}$ alloys, skutterudites, $\mathrm{Ag}-\mathrm{Pb}-\mathrm{Sb}-\mathrm{Te}$ or 'LAST', half-Heusler alloys and some high-ZT oxides. Certainly, there are also other useful approaches for enhancing TE properties, such as band structure engineering by doping $[17,20]$ and exploring new materials with complex crystalline structure [21,22], but such approaches are beyond the scope of the current review.

\section{Nanostructural approaches for enhancing thermoelectric performance}

The challenge to develop TE materials with superior performance is to tailor the interconnected physical parameters $\alpha, \sigma$ and $\kappa$ for a crystalline system. Nanostructures provide a chance to disconnect the linkage between thermal and electrical transport by introducing some new scattering mechanisms for $Z T$ enhancement. There are some excellent reviews focusing on nanocrystalline [26] and nanostructured thermoelectric materials [27] and interfaces in bulk thermoelectric materials [28]. Here, as illustrated in Figure 3, we attempt to categorize the morphological improvement of nanocomposites in TE materials in terms of several parameters, including dimension reduction, grain refinement and size reduction of a second phase. Single crystals usually present the best electrical conductivity because of the absence of grain boundaries that scatter charge carriers, but the $Z T$ values of such materials can be optimized only by adjusting the carrier concentration through elemental doping. Reducing the dimensions instead offers a new possibility to individually tune the TE parameters, as illustrated in Figure 3(a-d). When the system size decreases and approaches a scale comparable to the feature length of electron behavior (e.g. mean free path, wavelength) in any direction, the DOS is increased significantly due to quantum confinement [9], resulting in the enhancement of the Seebeck coefficient [29]. Meanwhile, the thermal conductivity is also reduced because the surface strongly scatters the propagation of phonons, as any dimension is less than the average free path of phonons. For example, $\mathrm{Bi}_{2} \mathrm{Te}_{3}$-based films prepared by pulsed laser deposition show $\kappa$ values of $0.3-0.4 \mathrm{~W} \mathrm{~m}^{-1} \mathrm{~K}^{-1}$, which are $25 \%$ lower than in the bulk [30]. It has also been reported that a silicon nanowire with a diameter of $10-20 \mathrm{~nm}$

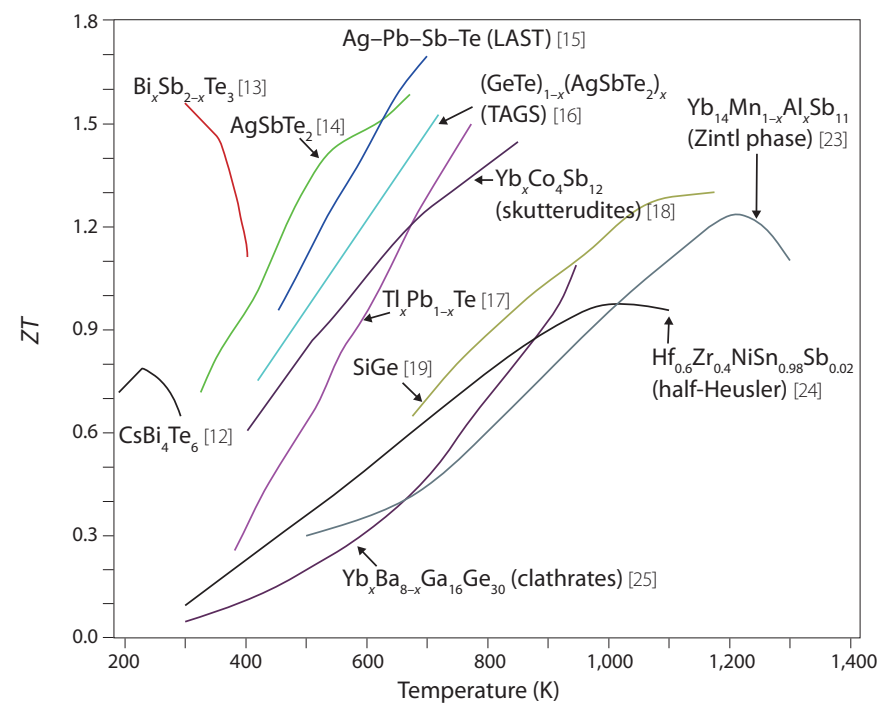

Figure 2. Figure of merit (ZT) of recent high-performance bulk thermoelectric materials as a function of applied temperature. The high performance of most of these materials is related to nanostructure engineering.

presents amazingly low thermal conductivity with a $\kappa_{\text {bulk }} / \kappa_{\text {nanowire }}$ ratio of as high as 25-150 near room temperature [31,32].

Apart from surfaces scattering, the thermal conductivity can be reduced significantly by introducing grain boundaries or interfaces, as illustrated in Figure $3(\mathrm{e}-\mathrm{h})$. However the suppression of lattice thermal conductivity $\left(\kappa_{\text {lat }}\right)$ due to boundaries will be overshadowed by the deterioration of carrier mobility $(\mu)$. Considering this trade-off, a nano/microcomposite TE material such as that shown in Figure 3(f) might provide better TE performance compared with monolithic coarse- or fine-grained samples [33,34]. In this nano/micro mix-grained composite, the dispersed nanoparticles are designed to scatter the phonon, while microparticles form a connected network for electron transport, and hence decouple the connection between thermal and electrical transport to a degree according to the percolation effect. Introducing an acoustically mismatched second phase in a matrix is another way to reduce the lattice thermal conductivity through an additional scattering mechanism [36].

Figure 3(i-l) shows the size reduction of isolated distinct phases or atoms in the composite, including sphere-, plate- and wire-shaped dispersed phases. Obvious $Z T$ improvements have been obtained in $(\mathrm{Zr}, \mathrm{Hf}) \mathrm{NiSn}$ half-Heusler alloys by adding nano- $\mathrm{ZrO}_{2}[36]$, in $\mathrm{Bi}_{2} \mathrm{Te}_{3}$ by adding nano-SiC particles [37], and in $\mathrm{Yb}_{y} \mathrm{Co}_{4} \mathrm{Sb}_{12}$ by dispersing in situ partially oxidized $\mathrm{Yb}_{2} \mathrm{O}_{3}$ nanoparticles [38]. It is also reported that more significant enhancements can be achieved by embedding metal or conductive nanoparticles into a TE material matrix: examples include lead and antimony in PbTe [39], antimony in $\mathrm{Yb}_{y} \mathrm{Co}_{4} \mathrm{Sb}_{12}$ [40], and ErAs in $\mathrm{In}_{0.53} \mathrm{Ga}_{0.47} \mathrm{As}$ [41]. This kind of nanoscale dispersed phase can be located at the grain boundary or within the grains. Shakouri et al. [42] calculated the effect of nanoparticle scattering on the power factor using a relaxationtime approximation, and validated the calculated model by comparing the results with the experimental data obtained for ErAs:InGaAlAs samples. They used the theory to maximize the power factor with respect to the volume content of nanoparticles and electron concentrations, as well as the barrier height. Chen et al. [43] developed a semiclassical electron transport model based on the Boltzmann transport equation to study the TE transport properties of nanocomposites. A relaxation-time model for electrical carrier-interface scattering was developed to account for the filtering effect of low-energy electrons on transport properties. After fitting the model to data for bulk TE alloys, yielding reasonable adjustment parameters for bulk alloys similar to handbook values, the model was further validated by comparing the modeled TE properties with recently reported values for p-type $\left(\mathrm{Bi}_{y} \mathrm{Sb}_{2-y} \mathrm{Te}_{3}\right)-\left(\mathrm{Bi}_{0.5} \mathrm{Sb}_{1.5} \mathrm{Te}_{3}\right)$ and 


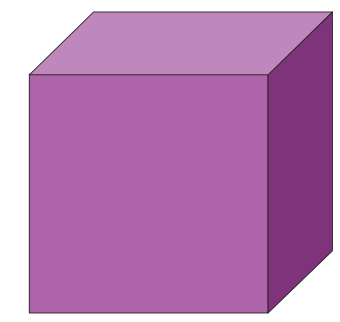

e

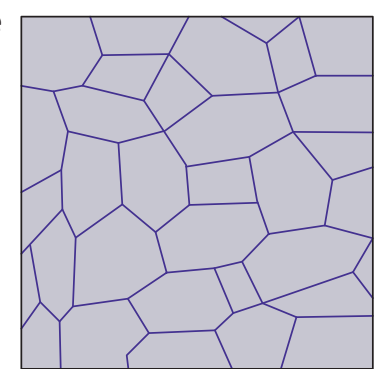

i

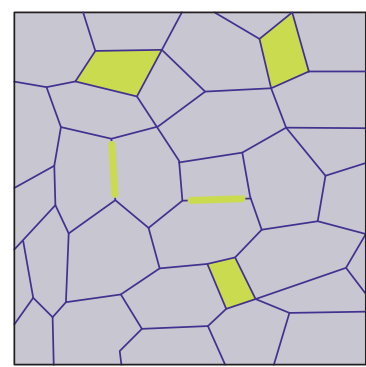

b

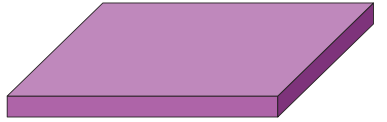

$\mathbf{f}$

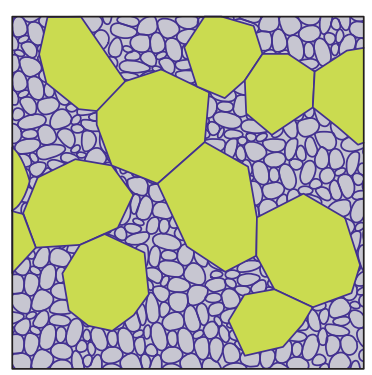

j

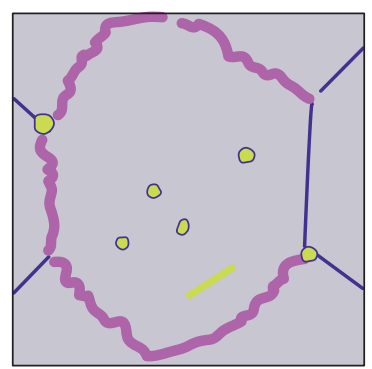

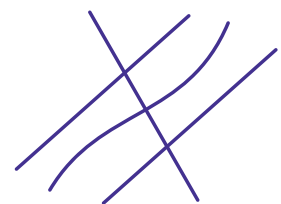

g

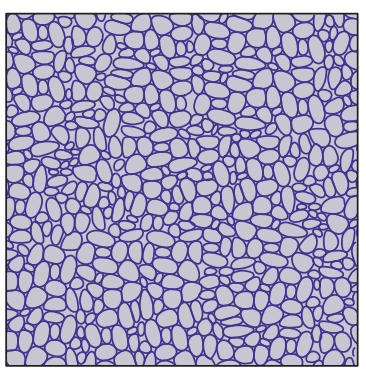

k

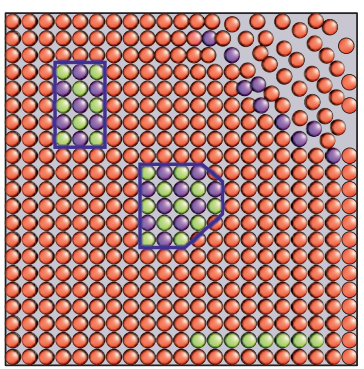

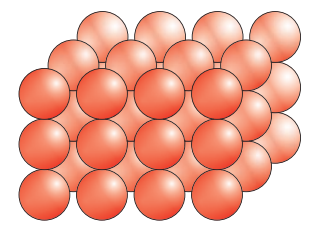

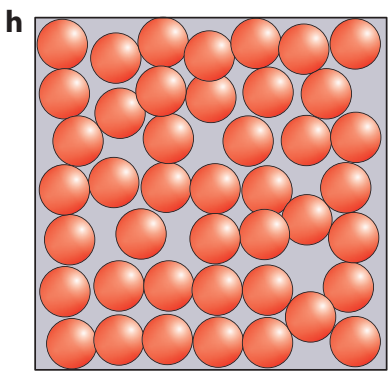

I

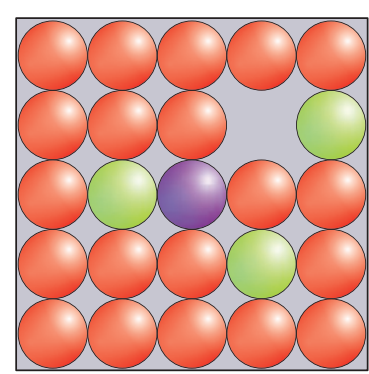

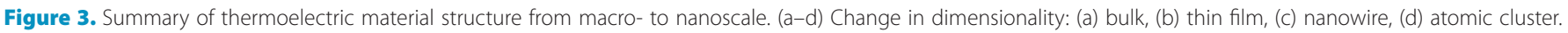

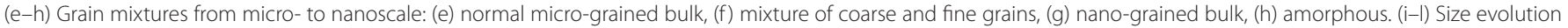

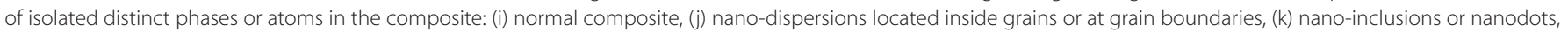
boundary modification, (I) atomic doping or alloying, and vacancies.

n-type $\left(\mathrm{Mg}_{2} \mathrm{Si}_{y} \mathrm{Ge}_{1-y}\right)-\left(\mathrm{Mg}_{2} \mathrm{Si}_{0.6} \mathrm{Ge}_{0.4}\right)$ nanocomposites. The inspired work of Kanatzidis's group revealed that a new kind of intrinsic nano-inclusion, as shown schematically in Figure $3(\mathrm{k})$, formatted by the segregation of silver and antimony in the lead sublattice of $\mathrm{PbTe}$, could achieve simultaneous phonon blocking and electron transmission.

Base on the same concept, Liu et al. [44] obtained a similar microstructure by partially substituting antimony with tellurium and tin in $\mathrm{CoSb}_{3}$, thereby obtaining a significant improvement. The theoretical study showed that nano-inclusions could reduce the thermal conductivity of the host medium below that of the alloying case, which is defined as an atomic random distribution [45], as shown in Figure 3(1). It can be noted through these examples that the combination of nanoengineering strategies has become a new trend, leading to such systems as a superlattice or quantum well wire combining reduced dimensionality with a second dispersion phase [46]. A current challenge in the development of nanocomposite TE materials is how to preserve the nanostructures within the bulk composite and prevent aggregation and interparticle growth. The solution to these issues significantly depends on the interfaces. A suitable interface could prohibit undesired grainboundary growth and interdiffusion, as well as selectively scattering phonons and charge carriers, potentially resulting in a considerably enhancement of $Z T$ values.

\section{Representative high-performance thermoelectric materials}

\section{$\mathrm{Bi}_{2} \mathrm{Te}_{3}$-based alloys}

$\mathrm{Bi}_{2} \mathrm{Te}_{3}$ has been studied extensively since 1954 [47] and is one of the most widely used TE materials. Nevertheless, this material still attracts plenty of interest, and has undergone significant enhancements as a
TE material. In 2008, Poudel et al. [48] reported a nano-composite $\mathrm{Bi}_{x} \mathrm{Sb}_{2-x} \mathrm{Te}_{3}$ bulk with $Z T$ of ca. 1.4 at $373 \mathrm{~K}$. This material was fabricated by mechanical milling of a commercial ingot into a nanopowder that was subsequently consolidated by hot pressing. Their microstructural analysis revealed that the high $Z T$ value is partially attributable to a significant decrease in $\kappa_{\text {lat }}$ due to scattering at the grain boundary and the presence of nano-precipitates [49]. Tang et al. [14] developed a melt-quench-anneal-spark plasma sintering method to form bulk nanostructural p-type $\mathrm{Bi}_{0.52} \mathrm{Sb}_{1.48} \mathrm{Te}_{3}$ with a $Z T$ value at of 1.56 at $300 \mathrm{~K}$. Cao et al. [50] obtained a maximum $Z T$ of 1.47 at ca. $438 \mathrm{~K}$ for $\mathrm{Bi}_{2} \mathrm{Te}_{3} / \mathrm{Sb}_{2} \mathrm{Te}_{3}$ bulk nanocomposites with laminated nanostructures using a simple route involving hydrothermal synthesis and hot pressing.

The nano/micro composite has now become an important direction for research. Recently, the author's group has conducted much work on $\mathrm{SiC} / \mathrm{Bi}_{2} \mathrm{Te}_{3}$ nano/microcomposite TE materials. Our studies have revealed that a $20 \%$ enhancement in $Z T$ can be achieved by adding $\mathrm{SiC}$ nanoparticles at just $0.2 \mathrm{vol} \%$. However, the $Z T$ value decreased quickly at higher $\mathrm{SiC}$ concentrations because $\mathrm{SiC}$ has much higher electrical resistivity and thermal conductivity compared to $\mathrm{Bi}_{2} \mathrm{Te}_{3}$ [51]. The addition of $\mathrm{SiC}$ also obviously enhanced the micro-hardness and fracture toughness, resulting in improved machining properties for device manufacturing, particularly for miniaturized devices [37].

However, a decrease in $\kappa_{\text {lat }}$ by nanoparticle dispersion also leads to a decrease in carrier mobility, and as a result, no more enhancement of $Z T$ is obtained. Recently, a percolation effect has been introduced to tune TE transport properties, and a proof-of-concept experiment has been conducted by Zhao et al. [34] In their experiment, two kinds of $\mathrm{Bi}_{2} \mathrm{Te}_{3}$ powders with large particles size difference (coarse, ca. $1 \mu \mathrm{m}$; fine, ca. $100 \mathrm{~nm}$ ) were fabricated by mechanical alloying, and mixtures 
a

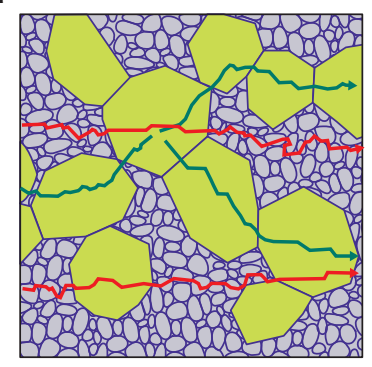

- Phonon - Carrier b

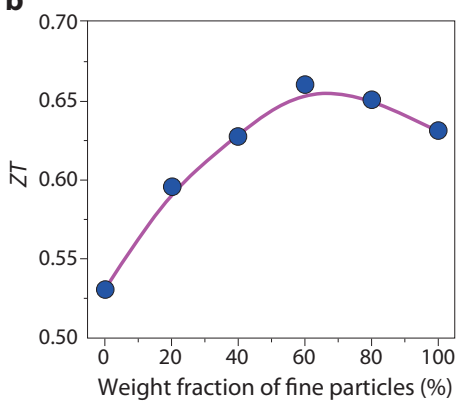

Figure 4. Illustration of the principle of percolation in nano/microcomposites. (a) Presumed carrier and phonon transport path in the composite. (b) Weight fraction dependence of thermoelectric $Z T$ values at room temperature in $\mathrm{Bi}_{2} \mathrm{Te}_{3}$ composite, Adapted from Ref. 34, reproduced with permission (@ 2009 AIP).

with various fine/coarse ratios were consolidated by spark plasma sintering. An enhanced $Z T$ value at room temperature was achieved with an optimized fine/coarse volume ratio of $6 / 4$.

The mechanism of TE enhancement by the percolation effect is illustrated in Figure 4(a). The charge carriers likely 'select' a path - the low-resistivity channel connected by the large grains - whereas the phonons do not choose their path. The system of channels via coarse grains would have low electrical resistivity and thermal conductivity if more fine particles are included. As the Seebeck coefficient does not change significantly by varying the grain size, the $Z T$ value could be tuned by adjusting the volume percentage of fine particles as shown in Figure 4(b). According to the principle of the percolation effect, a high coarse/fine size ratio in a powder would generate a more significant enhancement of $Z T$. The quantitative description can be given by $Z T=\alpha^{2} / L\left(1+\kappa_{\mathrm{l}} / \kappa_{\mathrm{e}}\right)$, where $L$ is the Lorenz number, $\kappa_{\mathrm{l}}$ is the lattice thermal conductivity and $\kappa_{\mathrm{e}}$ is the electrical thermal conductivity. Microstructural modifications allow the fundamental transport parameters (carrier mobility and lattice thermal conductivity) to be modified for improved TE performance. For example, carrier mobility can be increased by increasing the grain size, which reduces the density of grain boundaries and results in an increase in electrical conductivity. Low lattice thermal conductivity values can be obtained by grain boundary scattering. $Z T$ values will be enhanced by reducing the ratio of lattice thermal conductivity to electrical thermal conductivity $\left(\kappa_{1} / \kappa_{\mathrm{e}}\right)$ through optimization of the volume content of grain boundaries.

\section{$\mathrm{CoSb}_{3}$-based skutterudites}

The binary skutterudites possess a $\mathrm{CoAs}_{3}$-type structure with the general chemical formula $\mathrm{MX}_{3}$, where $\mathrm{M}$ is the transition-metal cobalt, rhodium or iridium, and $\mathrm{X}$ is phosphorus, arsenic or antimony [52,53], as shown in Figure 5(a). Among the skutterudite family, $\mathrm{CoSb}_{3}$ has received the most interest to day because of its higher weighted mobility $\left(m^{*}\right)^{3 / 2} \mu$ compared to other family members, where the $m^{*}$ and $\mu$ are the carrier effective mass and mobility. $\mathrm{CoSb}_{3}$ can have a very high power factor, but its high thermal conductivity $\left(10 \mathrm{~W} \mathrm{~m}^{-1} \mathrm{~K}^{-1}\right)$ prevents it from competing with state-of-the-art $\mathrm{Bi}_{2} \mathrm{Te}_{3}\left(1.0-1.5 \mathrm{~W} \mathrm{~m}^{-1} \mathrm{~K}^{-1}\right)$. However, this compound has a specific lattice structure with a large 'cage' located at the center of the unit cell, which could be filled with a small metal atom as shown in the Figure 5(a). Since the cage is much larger $(1.89 \AA)$ than most elemental ions, the filler is likely to rattle at the equilibrium position and hence generate significant scattering of phonons [6]. In 1996, Sales et al. [3] reported $\mathrm{Ce}_{0.9} \mathrm{Fe}_{3} \mathrm{CoSb}_{12}$ and $\mathrm{La}_{0.9} \mathrm{Fe}_{3} \mathrm{CoSb}_{12}$ with a remarkably low $\kappa_{\text {lat }}$ of $1.4 \mathrm{~W} \mathrm{~m}^{-1} \mathrm{~K}^{-1}$, one-seventh of that of unfilled $\mathrm{CoSb}_{3}$, and a consequently high $Z T$ of greater than 1.0. Recently, broad-ranging investigations have been carried out on $\mathrm{CoSb}_{3}$-based TE materials with respect to both doping strategies and synthesis processes, and some innovative results have been obtained. In 2001, Chen et al. [54] found that barium was a very good filling element, achieved a higher cage filling fraction (44\%) than lanthanum
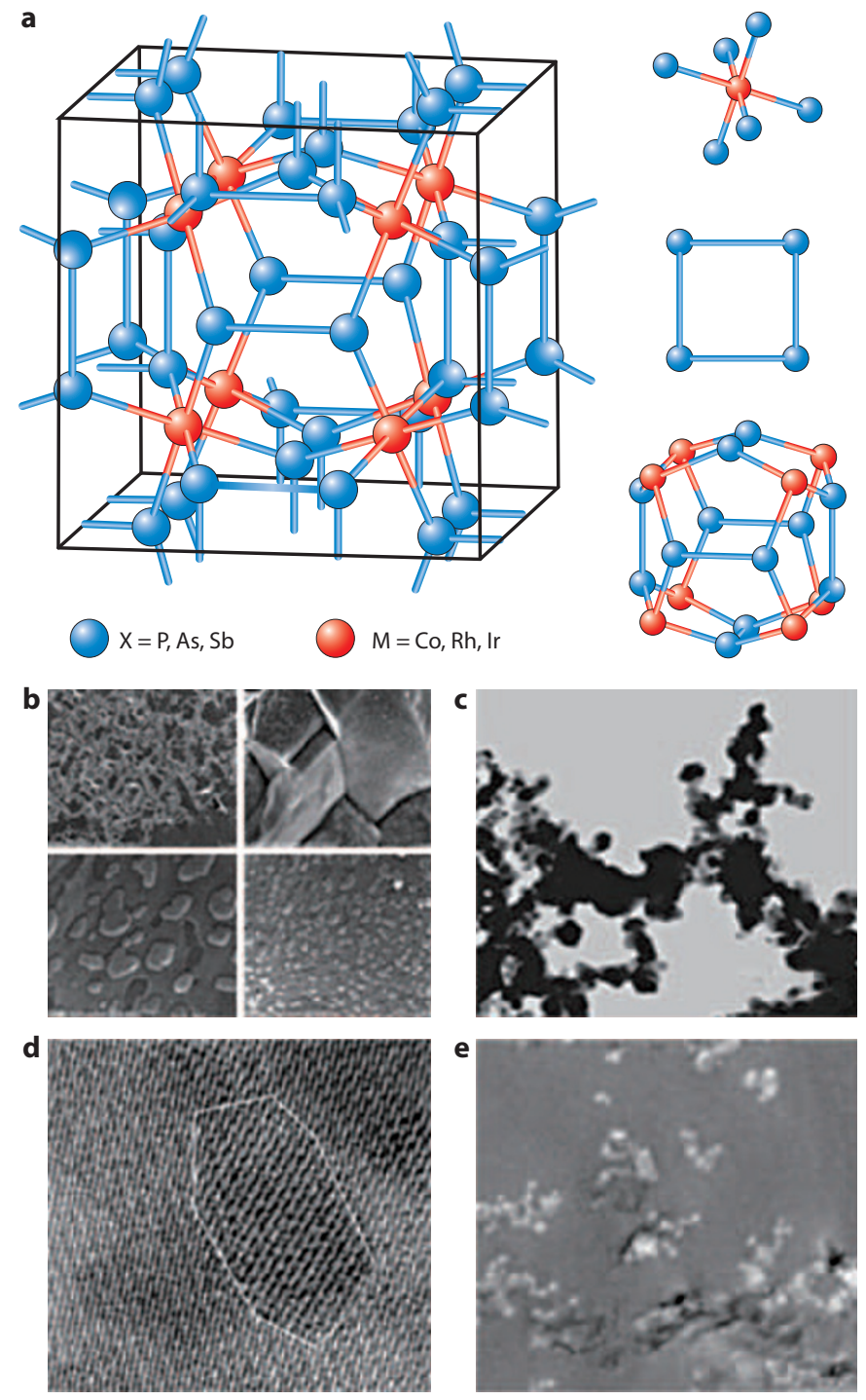

Figure 5. (a) Structure of skutterudites showing substructure details including the $\left[\mathrm{CoSb}_{6}\right]$ octahedron, $\left[\mathrm{Sb}_{4}\right]$ ring and $\left[\mathrm{CO}_{8} \mathrm{Sb}_{12}\right]$ dodecahedron. (b) InSb nanoparticles dispersed at grain boundaries. Modified after Ref. 58 (๔ 2009 AIP). (c) Nano-grained $\mathrm{CoSb}_{3}$ made by hydrothermal synthesis. Modified after Ref. 59 (๔ 2007 AIP). (d) Nanodots formed by segregation of tin and tellurium in the antimony sublattice of $\mathrm{CoSb}_{3}$. Modified after Ref. 44 (๔ 2008 ACS). (e) GaSb nano-inclusions dispersed in $\mathrm{Yb}_{0.26} \mathrm{CO}_{4} \mathrm{Sb}_{12}$ matrix. Modified after Ref. 18 (๔ 2010 Elsevier). All figures reproduced with permission.

(ca. 20\%) without charge compensation. Barium filling not only reduced $\kappa$, but also raised $\sigma$, leading to a high $Z T$ value in $\mathrm{Ba}_{y} \mathrm{Co}_{4} \mathrm{Sb}_{12}$. On the base of Chen's work, Tang et al. [55] systematically studied the system $\mathrm{R}_{y} \mathrm{M}_{x} \mathrm{Co}_{4-x} \mathrm{Sb}_{12}(\mathrm{R}=\mathrm{Ce}, \mathrm{Ba}, \mathrm{Y} ; \mathrm{M}=\mathrm{Fe}, \mathrm{Ni})$, and both $\mathrm{n}$ and p-type skutterudites with high performance were obtained. The p-type $\mathrm{Ce}_{0.28} \mathrm{Fe}_{1.5} \mathrm{Co}_{2.5} \mathrm{Sb}_{12}$ showed a peak $Z T$ of 1.1 at $750 \mathrm{~K}$, and n-type $\mathrm{Ba}_{0.30} \mathrm{Ni}_{0.05} \mathrm{Co}_{3.95} \mathrm{Sb}_{12}$ presented a maximum $Z T$ of 1.25 at $900 \mathrm{~K}$. From an industrial viewpoint, a good TE device requires the n-type and p-type legs to have similar mechanical and thermal properties in order to minimize the possibility of failure due to thermal stress. $\mathrm{CoSb}_{3}$ will be a very good choice for medium-temperature TE applications because both $\mathrm{n}$ - and p-type materials with high performance can be obtained in the same material system.

Theoretical research on skutterudites has at the same time made some important advancements. Shi et al. [56] studied the filling limit of filled skutterudites theoretically and experimentally. They found that the filling limit is related to the electronegativity difference between 

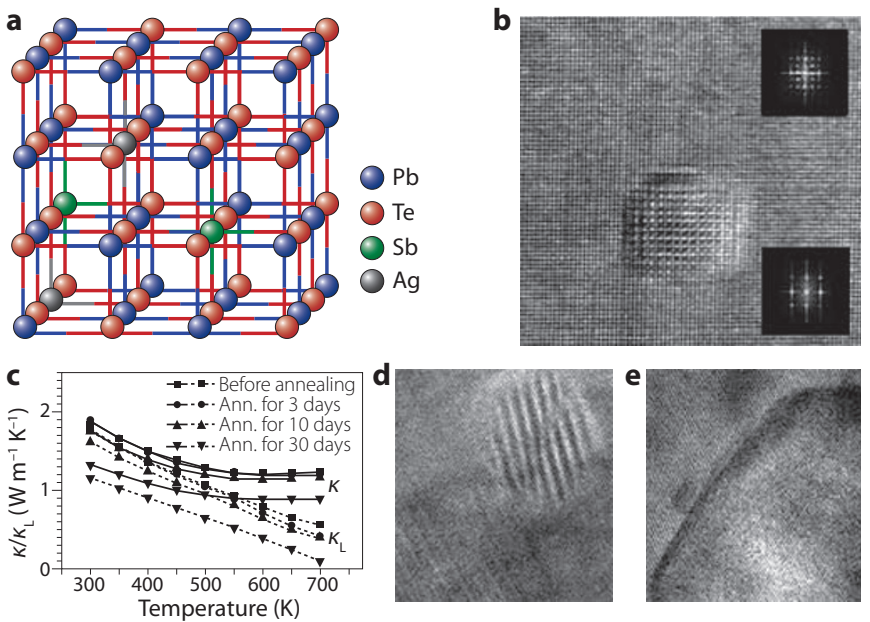

Figure 6. (a) Average Fm $\overline{3} m$ structure of LAST system. Adapted from Ref. 15 (๔ 2004 AAAS). (b) High-resolution image of a nano-inclusion in $\mathrm{Ag}_{0.53} \mathrm{~Pb}_{18} \mathrm{Sb}_{1.2} \mathrm{Te}_{20}$ taken along the [001] zone axis. Modified after Ref. 61 (๔ 2009 Wilegy-VCH). (c) Temperaturedependent thermal conductivity of MA-SPS-synthesized $\mathrm{Ag}_{0.8} \mathrm{~Pb}_{22} \mathrm{SbTe}_{20}$ after annealing. (d) Typical high-resolution transmission electron microscopy (HRTEM) image of an unannealed sample. (e) Typical HRTEM image of the sample after annealing for 30 days. (c-e) Adapted and modified after Ref. 64 (@ 2008 ACS). All figures reproduced with permission.

antimony and the filling element (R). Only the elements meeting the electronegativity difference condition of $\chi_{\mathrm{Sb}}-\chi_{\mathrm{R}}>0.8$ can enter the cage position of $\square \mathrm{Co}_{4} \mathrm{Sb}_{12}$ where $\square$ is the cage site. According to Shi's theoretical calculations, alkali elements could fill the cage position with a high filling fraction. In 2006, Pei et al. [57] successfully synthesized potassium-filled $\mathrm{CoSb}_{3}$, achieving a $Z T$ of ca. 1.0 at $800 \mathrm{~K}$ in the compound $\mathrm{K}_{0.38} \mathrm{Co}_{4} \mathrm{Sb}_{12}$. Recently, a breakthrough in filled skutterudites has been made by using a melt-quench method to fabricate an indium and cerium co-filled $\mathrm{CoSb}_{3}$ nanocomposite, for which a peak $Z T$ of 1.43 was obtained at $800 \mathrm{~K}$ (Figure 5(b)) [58]. The $\kappa_{\text {lat }}$ value of filled skutterudites is much lower than that of unfilled skutterudites, but still much higher than that of $\mathrm{Bi}_{2} \mathrm{Te}_{3}$-based TE compounds. Recent research suggests that reducing the grain size could be a fruitful route for the $\mathrm{CoSb}_{3}$-based TE materials. Hydrothermal synthesis is a versatile method for the fabrication of various nanoparticles. Zhao's group [59] explored this method to synthesize many TE nanoparticles, as shown in Figure 5(c). Bulk $\mathrm{CoSb}_{3}$ prepared by hydrothermal synthesis of nanopowder followed by spark plasma sintering or hot-pressing showed a $\kappa$ value of $1.61 \mathrm{~W} \mathrm{~m}^{-1} \mathrm{~K}^{-1}$ and a six-fold enhancement in $Z T$.

The author's group has used mechanical alloying and spark plasma sintering (MA-SPS) to fabricate pure $\mathrm{CoSb}_{3}$ with an average grain size of $100 \mathrm{~nm}$ and a $\kappa$ value of $2.92 \mathrm{~W} \mathrm{~m}^{-1} \mathrm{~K}^{-1}$ [60]. This value is one fourth of that of the single-crystal counterpart, and is even lower than that of some filled skutterudites. Further doping of this finegrained $\mathrm{CoSb}_{3}$ by partially co-substituting antimony with tin and tellurium raised the $Z T$ value to 1.1 at $820 \mathrm{~K}$, which is the highest value reported to date for unfilled skutterudites and is comparable to that of many filled skutterudites [44]. The remarkable benefit of codoping with tin and tellurium is a further decrease in lattice thermal conductivity compared with tellurium-doped $\mathrm{CoSb}_{3}$. The presence of sparse nanodots inside grains could also be responsible for the further decrease in lattice thermal conductivity, as shown in Figure 5(d). Nanocomposites, as an important route for tuning $\kappa$, have also been used in $\mathrm{CoSb}_{3}$-based TE materials. Chen's group [36] fabricated a composite containing ytterbium-filled $\mathrm{CoSb}_{3}$ and well-distributed $\mathrm{Yb}_{2} \mathrm{O}_{3}$ particles synthesized by in situ reaction. The $\mathrm{Yb}_{2} \mathrm{O}_{3}$ nanoparticles dispersed at the grain boundary and within the grains generate a combined effect that considerably scatters phonons but only slightly impacts on electrons, thereby providing a significant enhancement in peak $Z T$ to 1.3. Using a similar idea, as shown in Figure 6(e), the same research group has made a $(\mathrm{GaSb})_{0.2}-\mathrm{Yb}_{0.26} \mathrm{Co}_{4} \mathrm{Sb}_{12}$ nanocomposite exhibiting an improved $Z T$ value of up to 1.45 at $850 \mathrm{~K}$ [19].

\section{$\mathrm{Ag}_{n} \mathrm{~Pb}_{m} \mathrm{Sb}_{n} \mathrm{Te}_{m+2 n}$}

In 2004, Kanatzidis's group reported a high-performance TE material system with the general chemical formula of $\mathrm{Ag}_{1-x} \mathrm{~Pb}_{18} \mathrm{SbTe}_{20}$, termed LAST [15]. As shown in Figure 6(a), the LAST compounds possess an average $\mathrm{NaCl}$ structure ( $F m \overline{3} m$ symmetry), and could thus be considered to be antimony and silver co-doped PbTe. The same group used a melting plus extremely slow cooling method to develop a nanocomposite with many nanoscale inhomogeneities embedded in the $\mathrm{PbTe}$ matrix, as shown in Figure 6(b). These nano-inclusions are likely to be ideal phonon-blocking and electron-transmitting centers. Due to the special nanostructure, $\mathrm{Ag}_{1-x} \mathrm{~Pb}_{18} \mathrm{SbTe}_{20}$ showed a very high $Z T$ value of 2.2 at $800 \mathrm{~K}$. Although lower $Z T$ values of $1.5-1.7$ were confirmed in a subsequent report [62], the same study suggest that this serial compound is still the most competitive TE material. Kanatzidis's work has ignited broad research interest on the LAST system [14,62-65].

The author's group has been working on the synthesis of TE materials by MA-SPS since 2003, and has used this method successfully to fabricate LAST-system TE materials. Wang et al. [63] synthesized LAST compounds from lead, silver, antimony and tellurium elemental powders by MA-SPS at $673 \mathrm{~K}$, achieving a respectable $Z T$ value of 1.37 at $673 \mathrm{~K}$. They found that the TE properties of LAST compounds are very sensitive to chemical composition, particularly lead content. The optimized composition is $\mathrm{Ag}_{0.8} \mathrm{~Pb}_{22.5} \mathrm{SbTe}_{20}$ for the MA-SPS-synthesized sample, which is different from the composition $\left(\mathrm{Ag}_{0.8} \mathrm{~Pb}_{18} \mathrm{SbTe}_{20}\right)$ reported by Kanatzidis's group. Based on Wang's work, Zhou et al. [64] studied the effect of annealing treatment on the TE performance and microstructure of the LAST system and found that annealing treatment not only improved the electrical conductivity, it also reduced the thermal conductivity, as shown in Figure 6(c). Usually, it is extremely difficult to achieve an increase in electrical conductivity and a decrease in thermal conductivity simultaneously. Transmission electron microscopy analysis demonstrated that the annealing treatment assisted the formation of a nanostructure that favors electron transparent and phonon scattering, i.e. $\mathrm{Ag}_{\mathrm{Pb}}$ and $\mathrm{Sb}_{\mathrm{Pb}}$ coalesce to form intrinsic quantum nanodots, as shown in Figure $6(\mathrm{~d}-\mathrm{e})$. As a result, a significantly improved $Z T$ value of 1.5 was obtained at $703 \mathrm{~K}$. The LAST system can also be regarded as a nanocomposite with $\mathrm{AgPbTe}_{2}$ nano-phase dispersed in a PbTe matrix. Recently, Wang et al. [14] successfully synthesized bulk single-phase $\mathrm{AgSbTe}_{2}$ by MA-SPS. The material displayed an extremely low $\kappa$ value $\left(0.3 \mathrm{~W} \mathrm{~m}^{-1} \mathrm{~K}^{-1}\right)$ and high $Z T$ value of 1.59 at $673 \mathrm{~K}$.

Many studies have been carried out on the LAST system by Kanatzidis's group, particularly with respect to microstructure characterization [66] and exploration of p-type LAST systems [67-69]. They have found that a high $Z T$ value of 1.45 at $630 \mathrm{~K}$ is achievable in p-type $\operatorname{Ag}\left(\mathrm{Pb}_{1-x} \mathrm{Sn}_{x}\right)_{m} \mathrm{SbTe}_{2+m}$ by partially substituting the lead in the LAST system with tin [67]. Subsequent research has shown that simply replacing silver in the LAST system with sodium leads to a higher $Z T$ of 1.7 at $650 \mathrm{~K}$ in p-type $\mathrm{Na}_{0.95} \mathrm{~Pb}_{20} \mathrm{SbTe}_{22}$ [68]. More recently, potassium-based analogues of p-type $\mathrm{K}_{1-x} \mathrm{~Pb}_{m} \mathrm{Sb}_{\gamma} \mathrm{Te}_{m+2}$ materials have been reported. A maximum $Z T$ of 1.6 at $750 \mathrm{~K}$ has so far been achieved for a system with the composition $\mathrm{K}_{0.95} \mathrm{~Pb}_{20} \mathrm{Sb}_{1.2} \mathrm{Te}_{22}$ [69]. Both $\mathrm{n}$ - and p-type LAST materials can be obtained by adjusting the chemical composition, making the system is particularly promising for application in power generation.

\section{Half-Heusler compounds}

Compared with the PbTe or LAST systems, half-Heusler compounds are more environmentally benign and hence have attracted increasing levels of interest [70-74]. Half-Heusler compounds are crystallized in the $\mathrm{MgAgAs}$-type structure with space group $F \overline{4} 3 \mathrm{~m}$, which can be regarded as two interpenetrating cubic face-centered-cubic 

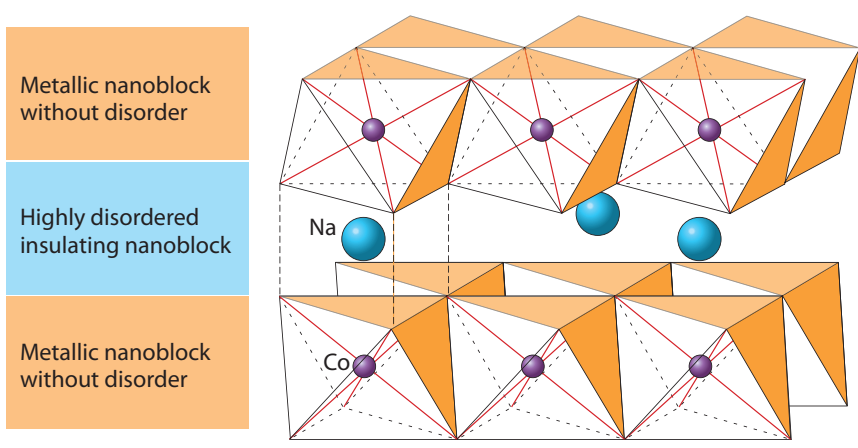

Figure 7. Crystal structure of $\mathrm{Na}_{x} \mathrm{CoO}_{2}$ (left) and the corresponding nanobolock structure (right) [81].

(FCC) motifs with a simple embedded cubic motif at the center [75]. Since half-Heusler compounds have a good combination of narrow band-gap and sharp slope of DOS near the Fermi level, a very high power factor is expected. However, on account of the relative high $\kappa$, the $Z T$ values of the half-Heusler compounds are still much lower than those of the state-of-the-art TE materials. As there are three sublattice positions in a half-Heusler compound, isoelectronic alloying on different sublattice positions is the most common approach to reducing $\kappa$. Uher et al. [70] found that the isoelectronic alloying of $\mathrm{Zr}_{0.5} \mathrm{Hf}_{0.5} \mathrm{NiSn}$ produced a higher $Z T$ value than that of either $\mathrm{ZrNiSn}$ or HfNiSn alone due to a reduction in thermal conductivity. Shen et al. [71] investigated the effect of partial substitution of nickel with palladium on the TE properties of $\mathrm{ZrNiSn}$-based half-Heusler compounds. It was shown that this substitution results in a significant reduction in thermal conductivity, leading to an improvement in $Z T$ to 0.7 at ca. $800 \mathrm{~K}$ for the composition $\mathrm{Zr}_{0.5} \mathrm{Hf}_{0.5} \mathrm{Ni}_{0.8} \mathrm{Pd}_{0.2} \mathrm{Sn}_{0.99} \mathrm{Sb}_{0.01}$. Later, Culp et al. [72] reported a slightly enhanced $Z T$ value of 0.8 at $800 \mathrm{~K}$ in $\mathrm{Hf}_{0.75} \mathrm{Zr}_{0.25} \mathrm{Ni}_{0.9} \mathrm{Pd}_{0.1} \mathrm{Sn}_{0.975} \mathrm{Sb}_{0.0025}$.

Since most of the current work on half-Heusler is focused on isoelectronic alloying, it is reasonable to anticipate that there is considerable room for further improvement in $Z T$ through the use of various nanostructures. Systematic theoretical calculations on 36 kinds of half-Heusler compounds by Yang et al. [75], demonstrates that there are potential new half-Heusler compounds beyond the well-studied n-type $\mathrm{MNiSn}$ ( $\mathrm{LaPdBi}, \mathrm{NdCoSn}, \mathrm{ZrCoBi}$, etc.) and p-type $\mathrm{MCoSb}$ (NdCoSn, TiCoSb, NdRhSb, etc.). Experimental investigations on these new compounds should be strongly encouraged.

\section{Other systems}

Oxide materials usually have high thermal stability and oxidization resistance, and are hence good for high-temperature applications. However, the extremely low electrical conductivity of these materials has resulted in them being almost entirely ignored for their potential utilization in the TE field. The discovery of $\mathrm{NaCo}_{2} \mathrm{O}_{4}$ as a promising TE material in 1997 [76] completely changed the traditional understanding of oxides. $\mathrm{NaCo}_{2} \mathrm{O}_{4}$ posses a similar structure to the high-temperature superconductor compound $\mathrm{YBaCuO}$, with a $\mathrm{CoO}_{2}$ layer and sodium layer constructing a laminated structure, as shown in Figure 7. $\mathrm{NaCo}_{2} \mathrm{O}_{4}$ has high electrical conductivity and a high Seebeck coefficient, resulting in a remarkably high power factor of $5,000 \mathrm{~W} \mathrm{~m}^{-1} \mathrm{~K}^{-2}$, even higher than that of state-of-the-art $\mathrm{Bi}_{2} \mathrm{Te}_{3}\left(4,000 \mathrm{~W} \mathrm{~m}^{-1} \mathrm{~K}^{-2}\right)$ [76]. The strong electronic correlation effect in this material has been attributed to the unusually large Seebeck coefficient [77]. Many researchers subsequently started to explore high-performance oxides for TE applications, and some promising TE oxides have emerged, including $\mathrm{Zn}_{1-x} \mathrm{Al}_{x} \mathrm{O}$ (n-type, $Z T \approx 0.30$ at $1273 \mathrm{~K}$ ) [78], $\mathrm{La}_{1-x} \mathrm{Sr}_{x} \mathrm{CoO}_{3}$ (p-type, $Z T \approx 0.18$ at room temperature) [79], and $\mathrm{La}_{1-x} \mathrm{Y}_{x} \mathrm{CuO}_{4}$ (p-type, $\mathrm{ZT} \approx 0.17$ at $330 \mathrm{~K})[80]$.

Recently, the concept of nanoblock integration into layer-structured hybrid crystal (natural superlattice) has been proposed by Japanese researchers $[81,82]$. According to this idea, the layered $\mathrm{NaCo}_{2} \mathrm{O}_{4}$ is regarded as a hybrid crystal of ordered $\mathrm{CoO}_{2}$ nanosheets and disordered sodium nanosheets. The $\mathrm{CoO}_{2}$ nanosheets form a strongly connected electron system that serves as an electronic transport layer, leading to a high power factor, whereas the sodium ion nanoblock layers or calcium cobalt oxide misfit layers act as phonon scattering regions to provide low thermal conductivity. The development of hybrid crystals or natural superlattices composed of a periodic arrangement of nanoblocks or nanosheets having different functions is a new direction for achieving high TE performance. On the basis of the idea of a hybrid crystal, a series of modulated layered natural superlattices have been synthesized, including $(\mathrm{Na}, \mathrm{Ca}) \mathrm{Co}_{2} \mathrm{O}_{4}[83],\left(\mathrm{Bi}_{1.79} \mathrm{Sr}_{1.98} \mathrm{O}_{y}\right)_{0.63}\left(\mathrm{RhO}_{2}\right)$ [84] and $(\mathrm{SrO})$ $\left(\mathrm{SrTiO}_{3}\right)_{m}$ [85]. Recently, Lwadaki et al. [86] successfully synthesized a material with a pseudo-one-dimensional structure, $\mathrm{Ba}_{3} \mathrm{Co}_{2} \mathrm{O}_{6}\left(\mathrm{CO}_{3}\right)_{0.7}$, similar to the $2 \mathrm{H}$-perovskite-type $\mathrm{BaCoO}_{3}$ and containing $\mathrm{CoO}_{6}$ octahedral columns running parallel to the crystallographic $c$ axis.

\section{Summary and outlook}

Thermoelectric conversion technology is receiving increasing attention because of the worldwide energy and environment problems, and great progress has been made in the development of high-performance TE materials, including bulk, film and nanowire materials. Nanostructures such as nano-dispersions and nanoscale heterogeneities have been found to be effective in reducing thermal conductivity to a greater degree than electrical conductivity, resulting in an enhanced figure of merit for the bulk thermoelectric material. However, additional approaches will likely be indispensable for increasing the power factor to achieve further significant $Z T$ improvements. Theoretical modeling studies are highly anticipated for designing nanostructured or nanocomposite thermoelectric materials with enhanced Seebeck coefficient based on carrier-energy filtering or quantum confinement effects. As the cost and environmental impact of TE materials must also be taken into account for non-specialized applications, TE compounds free of tellurium and lead will become increasingly attractive targets for research in the future.

\section{Acknowledgments}

The authors acknowledge the financial support of the National Basic Research Program of China (Grant no. 2007CB607500) and the Tsinghua University Initiative Scientific Research Program, as well as the National Nature Science Foundation (Grant no. 50820145203).

\section{References}

1. L. E. Bell, Science 321, 1457 (2008).

2. A. F. Loffe, Semiconductor Thermoelements and Thermoelectric Cooling (Infosearch, UK, 1957).

3. B. C. Sales, D. Mandrus, R. K. Williams, Science 272, 1325 (1996).

4. G. S. Nolas, D. T. Morelli, T. M. Tritt, Annu. Rev. Mater. Sci. 29, 89 (1999).

5. G. S. Nolas, J. L. Cohn, G. A. Slack, S. B. Schujman, Appl. Phys. Lett. 73, 178 (1998).

6. G. A. Slack, in CRC Handbook of Thermoelectrics, D. M. Rowe ed. (CRC Press, USA, 1995).

7. S. M. Kauzlarich, S. R. Brown, G. J. Snyder, Dalton T. 21, 2099 (2007)

8. E. S. Toberer, A. F. May, G. J. Snyder, Chem. Mater. 22, 624 (2010).

9. L. D. Hicks, M. S. Dresselhaus, Phys. Rev. B 47, 12727 (1993).

10. R. Venkatasubramanian, E. Siivola, T. Colpitts, B. O’Quinn, Nature 413, 597 (2001).

11. T. C. Harman, P. J. Taylor, M. P. Walsh, B. E. LaForge, Science 297, 2229 (2002).

12. D.-Y. Chung et al., Science 287, 1024 (2000).

13. W. Xie, X. Tang, Y. Yan, Q. Zhang, T. M. Tritt, Appl. Phys. Lett. 94, 102111 (2009).

14. H. Wang, J.-F. Li, M. Zou, T. Sui, Appl. Phys. Lett. 93, 202106 (2008).

15. K. F. Hsu et al., Science 303, 818 (2004).

16. S. H. Yang et al., Acs. Sym. Ser. 19, 245707 (2008).

17. J. P. Heremans et al., Science 321, 554 (2008).

18. Z. Xiong, X. Chen, X. Huang, S. Bai, L. Chen, Acta Mater. 58, 3995 (2010). 
19. X. W. Wang et al., Appl. Phys. Lett. 93, 193121 (2008).

20. W.-S. Liu, L.-D. Zhao, B.-P. Zhang, H.-L. Zhang, J.-F. Li, Appl. Phys. Lett. 93, 042109 (2008).

21. J.-S. Rhyee et al., Nature 459, 965 (2009).

22. G. J. Snyder, E. S. Toberer, Nat. Mater. 7, 105 ( 2008).

23. E. S. Toberer et al., Adv. Funct. Mater. 18, 2795 (2008).

24. C. Yu et al., Acta Mater. 57, 2757 (2009).

25. X. Tang, P. Li, S. Deng, Q. Zhang, J. Appl. Phys 104, 013706 (2008).

26. H. Gleiter, Prog. Mater. Sci. 33, 223 (1989).

27. P. Pichanusakorn, P. Bandaru, Mater. Sci. Eng. R 67, 19 (2010).

28. D. L. Medlin, G. J. Snyder, Curr. Opin. Colloid \& In. 14, 226 (2009).

29. H. Ohta et al., Nat. Mater. 6, 129 (2007)

30. R. S. Makala, K. Jagannadham, B. C. Sales, J. Appl. Phys 94, 3907 (2003).

31. A. I. Hochbaum, et al., Nature 451, 163 (2008).

32. A. I. Boukai et al., Nature 451, 168 (2008).

33. J. L. Mi, X. B. Zhao, T. J. Zhu, J. P. Tu, Appl. Phys. Lett. 91, 172116 (2007).

34. L.-D. Zhao, B.-P. Zhang, W.-S. Liu, J.-F. Li, J. Appl. Phys 105, 023704 (2009).

35. D. G. Cahill et al., J. Appl. Phys 93, 793 (2003).

36. L. D. Chen, X. Y. Huang, M. Zhou, X. Shi, W. B. Zhang, J. Appl. Phys 99, 064305 (2006)

37. L.-D. Zhao et al., J. Alloy. Compd. 455, 259 (2008).

38. X. Y. Zhao et al., J. Appl. Phys 99, 053711 (2006).

39. J. R. Sootsman et al., Angew. Chem. Int. Ed. 47, 8618 (2008).

40. H. Li, X. Tang, X. Su, Q. Zhang, Appl. Phys. Lett. 92, 202114 (2008).

41. W. Kim et al., Phys. Rev. Lett. 96, 045901 (2006).

42. M. Zebarjadi et al., Appl. Phys. Lett. 94, 202105 (2009).

43. J. Zhou, X. Li, G. Chen, R. Yang Phys. Rev. B 82, 115308 (2010).

44. W.-S. Liu, B.-P. Zhang, L.-D. Zhao, J.-F. Li, Chem. Mater. 20, 7526 (2008).

45. N. Mingo, D. Hauser, N. P. Kobayashi, M. Plissonnier, A. Shakouri, Nano Lett. 9, 711 (2009)

46. M. S. Dresselhaus et al., in Recent Trends in Thermoelectric Materials Research: Part Three in Semiconduct. Semimet., Vol. 71, T. M. Tritt ed. (Academic, USA, 2001).

47. H. J. Goldsmid, R. W. Douglas, Brit. J. Appl. Phys 5, 386 (1954).

48. B. Poudel et al., Science 320, 634 (2008)

49. Y. Lan et al, Nano Lett. 9, 1419 (2009).

50. Y. Q. Cao, X. B. Zhao, T. J. Zhu, X. B. Zhang, J. P. Tu, Appl. Phys. Lett. 92, 143106 (2008).

51. J.-F. Li, J. Liu, Phys. Status Solidi A 203, 3768 (2006).

52. C. Uher, in Recent Trends in Thermoelectric Material Search I in Semiconduct. Semimet. Vol. 69, T. M. Tritt ed. (Academic, USA, 2001).
53. D. Bérardan et al., J. Appl. Phys 98, 033710 (2005).

54. L. D. Chen et al., J. Appl. Phys 90, 1864 (2001).

55. X. Tang, Q. Zhang, L. Chen, T. Goto, T. Hirai, J. Appl. Phys 97, 093712 (2005).

56. X. Shi, W. Zhang, L. D. Chen, J. Yang, Phys. Rev. Lett. 95, 185503 (2005).

57. Y. Z. Pei et al., Appl. Phys. Lett. 89, 221107 (2006).

58. H. Li, X. Tang, Q. Zhang, C. Uher, Appl. Phys. Lett. 94, 102114 (2009).

59. J. L. Mi, T. J. Zhu, X. B. Zhao, J. Ma, J. Appl. Phys 101, 054314 (2007).

60. W.-S. Liu, B.-P. Zhang, J.-F. Li, L.-D. Zhao, J. Phys D Appl. Phys 40, 566 (2007).

61. B. A. Cook et al., Adv. Funct. Mater. 19, 1254 (2009).

62. N. Chen et al., Appl. Phys. Lett. 87, 171903 (2005).

63. H. Wang et al., Appl. Phys. Lett. 88, 092104 (2006).

64. M. Zhou, J.-F. Li, T. Kita, J. Am. Chem. Soc. 130, 4527 (2008).

65. K. F. Cai et al., J. Alloy. Compd. 469,499 (2009).

66. E. Quarez et al., J. Am. Chem. Soc. 127, 9177 (2005).

67. J. Androulakis et al., Adv. Mater. 18, 1170 (2006).

68. P. F. P. Poudeu et al., Angew. Chem. Int. Ed. 45, 3835 (2006).

69. P. F. P. Poudeu, A. Guéguen, C.-I. Wu, T. Hogan, M. G. Kanatzidis, Chem. Mater. 22, 1046 (2010).

70. C. Uher, J. Yang, S. Hu, D. T. Morelli, G. P. Meisner, Phys. Rev. B 59, 8615 (1999).

71. Q. Shen et al., Appl. Phys. Lett. 79, 4165 (2001).

72. S. R. Culp, S. J. Poon, N. Hickman, T. M. Tritt, J. Blumm Appl. Phys. Lett. 88, 042106 (2006)

73. M. Zou, J.-F. Li, B. Du, D. Liu, T. Kita, J. Solid State Chem. 182, 3138 (2009).

74. M. Zhou, L. Chen, C. Feng, D. Wang, J.-F. Li, J. Appl. Phys 101, 113714 (2007).

75. J. Yang et al., Adv. Funct. Mater. 18, 2880 (2008).

76. I. Terasaki, Y. Sasago, K. Uchinokura, Phys. Rev. B 56, R12685 (1997).

77. Y. Ando, N. Miyamoto, K. Segawa, T. Kawata, I. Terasaki, Phys. Rev. B 60, 10580 (1999).

78. M. Ohtaki, T. Tsubota, K. Eguchi, H. Arai, J. Appl. Phys 79, 1816 (1996).

79. J. Androulakis, P. Migiakis, J. Giapintzakis, Appl. Phys. Lett. 84, 1099 (2004).

80. Y. Liu et al., J. Am. Ceram. Soc. 92, 934 (2009).

81. K. Koumoto, et al., in CRC thermoelectric handbook: Micro to Nano, D. M. Rowe ed. (CRC Press, USA, 2006).

82. K. Koumoto, I. Terasaki, R. Funahashi, MRS Bulletin 31, 206 (2006).

83. K. Takahata, Y. Iguchi, D. Tanaka, T. Itoh, Phys. Rev. B 61, 12551 (2000).

84. K. Yubuta, S. Okada, Y. Miyazaki, I. Terasaki, T. Kajitani, Jpn J. Appl. Phys 44, 8557 (2005).

85. K. H. Lee, S. W. Kim, H. Ohta, K. Koumoto, J. Appl. Phys 100, 063717 (2006).

86. K. Iwasaki et al., J. Appl. Phys 106, 034905 (2009).

\section{Author profiles}

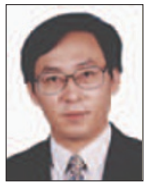

\section{Jing-Feng Li}

Jing-Feng Li is a professor of Materials Science and Engineering at Tsinghua University, China. He is also a subject editor for the Journal of Materials Processing Technology and is a member of the advisory board of NPG Asia Materials. He received his master and doctor degrees from Tohoku University, Japan, in 1988 and 1991, and his Bachelor of Engineering degree from the Huazhong University of Science and Technology, China, in 1984. He worked at Tohoku University as an assistant professor from 1992 to 1997, and as an associate professor from 1997 to 2002. Since joining Tsinghua University as full professor in 2002, he has been awarded the Outstanding Young Scientist award from the National Science Foundation, China (2003) and a Changjiang Scholar Professorship (2009).

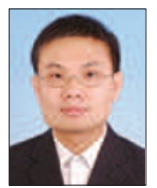

\section{Wei-Shu Liu}

Wei-Shu Liu is a postdoctoral research follow in the Department of Physics at Boston College, USA. He received his BS degree in material science and engineering from Chongqing University, China, and a PhD degree in the same field from the University of Science and Technology Beijing, China. He completed his doctoral thesis as a member of Jing-Feng Li's group from 2003 to 2008. In 2009, he spent a year as a postdoctoral research associate at the University of Washington, USA. His main research interests focus on the design, fabrication and tailoring of nanostructures for thermoelectric conversion applications.

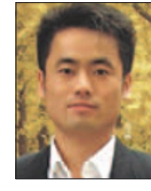

\section{Li-Dong Zhao}

Li-Dong Zhao is a postdoctoral research follow of the Institut de Chimie Moléculaire et des Matériaux d'Orsay at the Universite Paris Sud XI, France. He received his BS and MS degrees in material science from Liaoning Technical University, China, and his PhD degree in the same field from the University of Science and Technology Beijing, China. He completed his doctoral thesis as a member of Jing-Feng Li's group from 2005 to 2008. His research interests include the fabrication and properties of thermoelectric materials and layered structural superconductive materials.

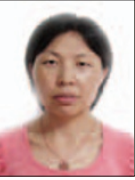

\section{Min Zhou}

Min Zhou is an associate professor in the Technical Institute of Physics and Chemistry, Chinese Academy of Sciences, China. She completed her postdoctoral research as a member of Jing-Feng Li's group in 2005. She received her PhD degree in material science and engineering from the Shanghai Institute of Ceramics, Chinese Academy of Sciences, China. Her main research interests focus on thermoelectric materials and their application in the field of energy conversion. 